POS PROCEEDINGS

\title{
Pion-pion scattering phase shifts with the stochastic LapH method
}

\author{
Brendan Fahy ${ }^{* a, b}$, John Bulava ${ }^{c}$, Ben Hörz ${ }^{c}$, Keisuke J. Juge ${ }^{d}$, Colin Morningstar $^{a}$, \\ and Chik Him Wonge \\ ${ }^{a}$ Dept. of Physics, Carnegie Mellon University, Pittsburgh, PA 15213, USA \\ ${ }^{b}$ High Energy Accelerator Research Organization (KEK), Ibaraki 305-0801, Japan \\ ${ }^{c}$ School of Mathematics, Trinity College, Dublin 2, Ireland \\ ${ }^{d}$ Dept. of Physics, University of the Pacific, Stockton, CA 95211, USA \\ ${ }^{e}$ Department of Physics, University of Wuppertal, Gaussstrasse 20, D-42119, Germany
}

Progress in calculating scattering phase shifts on $N_{f}=2+1$ anisotropic clover Wilson lattices is described. The stochastic LapH method facilitates computations in large volumes and for light pion masses. Results for pion masses down to $240 \mathrm{MeV}$, keeping $m_{\pi} L>4$, are presented.

The 32nd International Symposium on Lattice Field Theory,

23-28 June, 2014

Columbia University New York, NY

${ }^{*}$ Speaker. 


\section{Introduction}

Monte Carlo calculations in lattice QCD are necessarily carried out in finite volume. However, most of the excited hadrons we seek to study are unstable resonances. In finite volume with particular boundary conditions, the eigenvalues of the Hamiltonian are discrete since only certain momenta are allowed in order to satisfy the boundary conditions. Diagonalization of the Hamiltonian leads to a knowledge of the discrete stationary states. In infinite volume, a continuum of momenta are available and unstable excited hadrons decay to multi-hadron asymptotic states. In finite volume, there are no decays; instead, there is only quantum mechanical mixing between Fock states. Fortunately, it is possible to study the excited resonances using finite-volume calculations.

The idea that finite-volume energies can be related to infinite-volume scattering processes is actually rather old, dating back to Refs. [1,2] in the mid-1950s. Details on how to utilize such relationships in lattice QCD were first spelled out in Refs. [3, 4]. These calculations were later revisited using an entirely field theoretic approach in Ref. [5], and subsequent works have generalized their results to treat multi-channels with different particle masses and nonzero spins.

In the work described in this talk, we use a variety of two-pion energies in finite volume with different total momenta to calculate the $P$-wave scattering phase shifts in the $I=1 \mathrm{channel}$, and extract the mass and width of the $\rho$ resonance. Our preliminary results are obtained on a $32^{3} \times 256$ anisotropic lattice with quark masses tuned to yield a pion mass around $240 \mathrm{MeV}$. All needed Wick contractions are efficiently evaluated using a stochastic method[6] of treating the low-lying modes of quark propagation that exploits Laplacian Heaviside quark-field smearing.

\section{Scattering phase shifts from finite-volume energies}

For a given total momentum $\boldsymbol{P}=(2 \pi / L) \boldsymbol{d}$ in a spatial $L^{3}$ volume with periodic boundary conditions, where $\boldsymbol{d}$ is a vector of integers, we determine the total energy $E$ in the lab frame for a particular two-particle interacting state in our lattice QCD simulations. If the masses of the two particles are $m_{1}$ and $m_{2}$, we then boost to the center-of-mass frame and define the following quantities:

$$
\begin{aligned}
E_{\mathrm{cm}} & =\sqrt{E^{2}-\boldsymbol{P}^{2}}, \quad \gamma=\frac{E}{E_{\mathrm{cm}}}, \quad \boldsymbol{q}_{\mathrm{cm}}^{2}=\frac{1}{4} E_{\mathrm{cm}}^{2}-\frac{1}{2}\left(m_{1}^{2}+m_{2}^{2}\right)+\frac{\left(m_{1}^{2}-m_{2}^{2}\right)^{2}}{4 E_{\mathrm{cm}}^{2}}, \\
u^{2} & =\frac{L^{2} \boldsymbol{q}_{\mathrm{cm}}^{2}}{(2 \pi)^{2}}, \quad \boldsymbol{s}=\left(1+\frac{\left(m_{1}^{2}-m_{2}^{2}\right)}{E_{\mathrm{cm}}^{2}}\right) \boldsymbol{d} .
\end{aligned}
$$

The relationship between the finite-volume two-particle energy $E$ and the infinite-volume scattering amplitudes (and phase shifts) is encoded in the matrix equation:

$$
\operatorname{det}\left[1+F^{(\boldsymbol{s}, \gamma, u)}(S-1)\right]=0,
$$

where $S$ is the usual $S$-matrix whose elements can be written in terms of the scattering phase shifts, and the $F$ matrix is given in the $J L S$ basis states by

$$
F_{J^{\prime} m_{J^{\prime}} L^{\prime} S^{\prime} a^{\prime} ; J m_{J} L S a}^{(\boldsymbol{s}, \gamma, u}=\frac{\rho_{a}}{2} \delta_{a^{\prime} a} \delta_{S^{\prime} S}\left\{\delta_{J^{\prime} J} \delta_{m_{J^{\prime}} m_{J}} \delta_{L^{\prime} L}+W_{L^{\prime} m_{L^{\prime}} ; L m_{L}}^{\left(\boldsymbol{s}, \gamma,{ }^{\prime}, J^{\prime} m_{J^{\prime}}\right.}\left|L^{\prime} m_{L^{\prime}}, S m_{S}\right\rangle\left\langle L m_{L}, S m_{S} \mid J m_{J}\right\rangle\right\},
$$




\begin{tabular}{|ccc|}
\hline $\boldsymbol{d}$ & $\Lambda$ & $\cot \delta_{1}$ \\
\hline \hline$(0,0,0)$ & $T_{1 u}^{+}$ & $\operatorname{Re} w_{0,0}$ \\
$(0,0,1)$ & $A_{1}^{+}$ & $\operatorname{Re} w_{0,0}+\frac{2}{\sqrt{5}} \operatorname{Re} w_{2,0}$ \\
& $E^{+}$ & $\operatorname{Re} w_{0,0}-\frac{1}{\sqrt{5}} \operatorname{Re} w_{2,0}$ \\
$(0,1,1)$ & $A_{1}^{+}$ & $\operatorname{Re} w_{0,0}+\frac{1}{2 \sqrt{5}} \operatorname{Re} w_{2,0}-\sqrt{\frac{6}{5}} \operatorname{Im} w_{2,1}-\sqrt{\frac{3}{10}} \operatorname{Re} w_{2,2}$, \\
& $B_{1}^{+}$ & $\operatorname{Re} w_{0,0}-\frac{1}{\sqrt{5}} \operatorname{Re} w_{2,0}+\sqrt{\frac{6}{5}} \operatorname{Re} w_{2,2}$, \\
& $B_{2}^{+}$ & $\operatorname{Re} w_{0,0}+\frac{1}{2 \sqrt{5}} \operatorname{Re} w_{2,0}+\sqrt{\frac{6}{5}} \operatorname{Im} w_{2,1}-\sqrt{\frac{3}{10}} \operatorname{Re} w_{2,2}$ \\
$(1,1,1)$ & $A_{1}^{+}$ & $\operatorname{Re} w_{0,0}+2 \sqrt{\frac{6}{5}} \operatorname{Im} w_{2,2}$ \\
& $E^{+}$ & $\operatorname{Re} w_{0,0}-\sqrt{\frac{6}{5}} \operatorname{Im} w_{2,2}$ \\
\hline
\end{tabular}

Table 1: Expressions for the $P$-wave phase shifts $\delta_{1}\left(E_{\mathrm{cm}}\right)$ relevant for $I=1 \pi \pi$ scattering for various $\boldsymbol{d}$ and irreps $\Lambda$. The quantities $w_{l m}$ are defined in Eq. (2.11). The irrep labels are discussed in Ref. [7].

where $J, J^{\prime}$ refer to total angular momentum, $L, L^{\prime}$ are total orbital angular momenta, $S, S^{\prime}$ refer to total intrinsic spin in the above equation, $a, a^{\prime}$ label channels, $\rho_{a}=1$ for distinguishable particles and $\rho_{a}=\frac{1}{2}$ for identical particles, and

$$
W_{L^{\prime} m_{L^{\prime}} ; m_{L}}^{(\boldsymbol{s}, \gamma, u)}=\frac{2 i}{\pi \gamma u^{l+1}} \mathscr{Z}_{l m}\left(\boldsymbol{s}, \gamma, u^{2}\right) \int d^{2} \Omega Y_{L^{\prime} m_{L^{\prime}}}^{*}(\Omega) Y_{l m}^{*}(\Omega) Y_{L m_{L}}(\Omega) .
$$

Notice that $F^{(s, \gamma, u)}$ is diagonal in channel space, but mixes different total angular momentum sectors, whereas $S$ is diagonal in angular momentum, but has off-diagonal elements in channel space. Also, the matrix elements of $F^{(\boldsymbol{s}, \gamma, u)}$ depend on the total momentum $\boldsymbol{P}$ through $\boldsymbol{s}$, whereas the matrix elements of $S$ do not. The Rummukainen-Gottlieb-Lüscher (RGL) shifted zeta functions are evaluated using

$$
\begin{aligned}
\mathscr{Z}_{l m}\left(\boldsymbol{s}, \gamma, u^{2}\right) & =\sum_{\boldsymbol{n} \in \mathbb{Z}^{3}} \frac{\mathscr{Y}_{l m}(z)}{\left(z^{2}-u^{2}\right)} e^{-\Lambda\left(z^{2}-u^{2}\right)}+\delta_{l 0} \gamma \pi e^{\Lambda u^{2}}\left(2 u D(u \sqrt{\Lambda})-\Lambda^{-1 / 2}\right) \\
& +\frac{i^{l} \gamma}{\Lambda^{l+1 / 2}} \int_{0}^{1} d t\left(\frac{\pi}{t}\right)^{l+3 / 2} e^{\Lambda t u^{2}} \sum_{\substack{\boldsymbol{n} \in \mathbb{Z}^{3} \\
\boldsymbol{n} \neq 0}} e^{\pi i \boldsymbol{n} \cdot \boldsymbol{s}} \mathscr{Y}_{l m}(\boldsymbol{w}) e^{-\pi^{2} \boldsymbol{w}^{2} /(t \Lambda)},
\end{aligned}
$$

where $\boldsymbol{z}=\boldsymbol{n}-\boldsymbol{\gamma}^{-1}\left[\frac{1}{2}+(\gamma-1) s^{-2} \boldsymbol{n} \cdot \boldsymbol{s}\right] \boldsymbol{s}$ and $\boldsymbol{w}=\boldsymbol{n}-(1-\gamma) s^{-2} \boldsymbol{s} \cdot \boldsymbol{n} \boldsymbol{s}$, the spherical harmonic polynomials are given by $\mathscr{Y}_{l m}(\boldsymbol{x})=|\boldsymbol{x}|^{l} Y_{l m}(\widehat{\boldsymbol{x}})$, and $D(x)$ is the Dawson function, defined by

$$
D(x)=e^{-x^{2}} \int_{0}^{x} d t e^{t^{2}}
$$

We choose $\Lambda \approx 1$, although the final answer is independent of this choice. Choosing $\Lambda$ near unity allows sufficient convergence speed of the summations. Gauss-Legendre quadrature is used to perform the integral, and the Dawson function is evaluated using a Rybicki approximation.

The scattering processes we study conserve both total angular momentum $J$ and the projection of total angular momentum, say $M_{J}$. Given orthonormal states, then the unitarity of the $S$-matrix tells us that

$$
\left\langle J^{\prime} m_{J^{\prime}}^{\prime} L^{\prime} S^{\prime} a^{\prime}|S| J m_{J} L S a\right\rangle=\delta_{J^{\prime} J} \delta_{m_{J^{\prime}} m_{J}} s_{L^{\prime} S^{\prime} a^{\prime}, L S a}^{(J)}(E),
$$



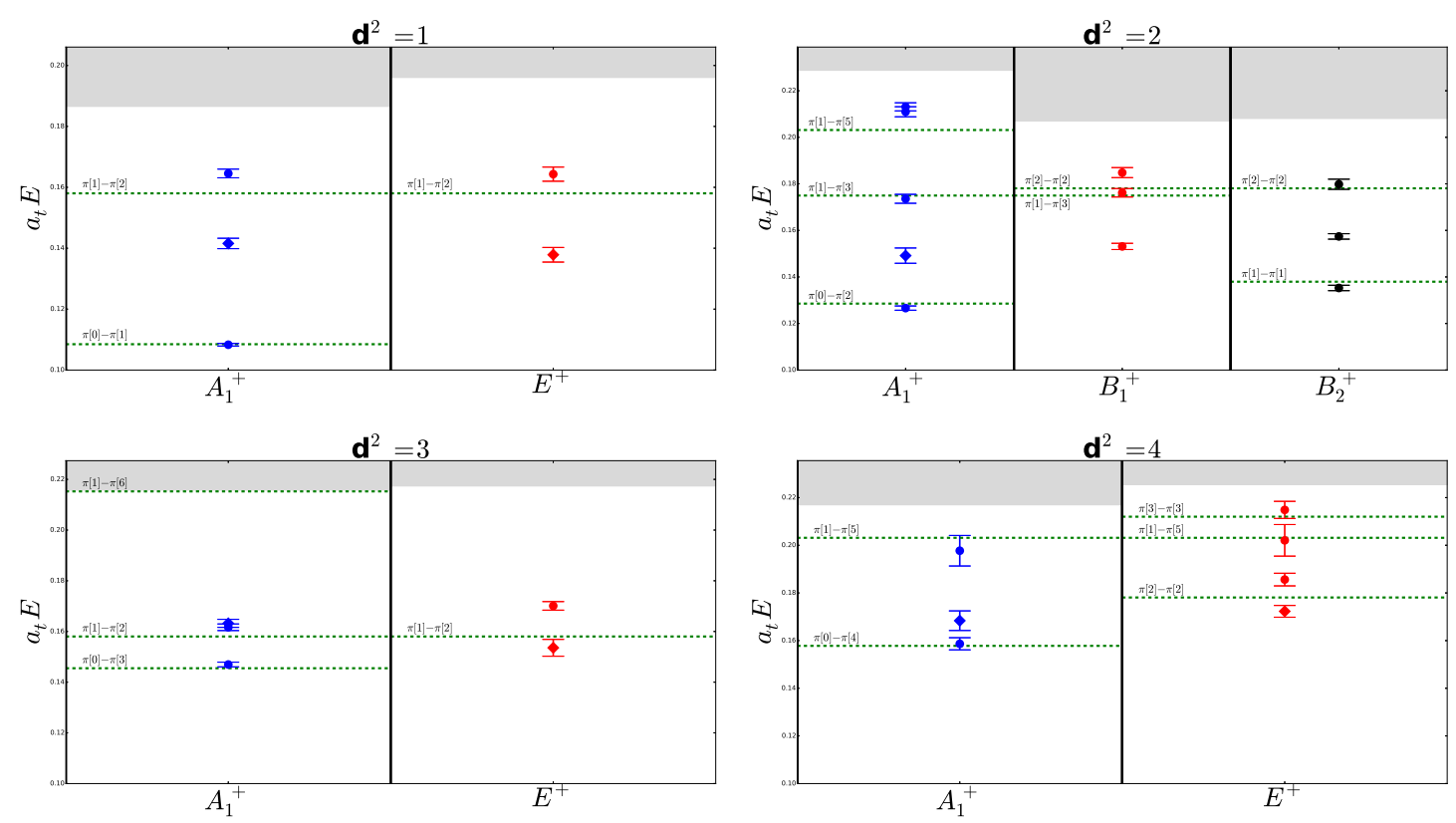

Figure 1: Energies $a_{t} E$ of $\pi \pi$ states for various $\boldsymbol{d}^{2}$. Dashed lines indicate the non-interacting energies of allowed $\pi \pi$ states. The shaded region indicates the inelastic thresholds. Diamond markers indicate levels with large overlaps with the $q \bar{q}$ operator corresponding to the $\rho$ meson.

where $a^{\prime}, a$ denote other defining quantum numbers, such as channel, and $s^{(J)}$ is a unitary matrix that is independent of $m_{J}$ due to rotational invariance. If the two particles have zero spin $s_{1}=s_{2}=0$ and there is only one channel, then

$$
s^{(J)}=s^{(L)}=e^{2 i \delta_{L}(E)},
$$

where $\delta_{L}(E)$ are known as the scattering phase shifts. The factor of 2 is conventional to agree with a certain definition when scattering from a central potential.

For single-channel $\pi \pi$ scattering, $s_{1}=s_{2}=0$, so $S=0$ and $J=L$, in which case Eq. (2.4) simplifies to

$$
F_{L^{\prime} m_{L^{\prime}} ; L m_{L}}^{(\boldsymbol{s}, \gamma, j)}=\frac{1}{2}\left(\delta_{L^{\prime} L} \delta_{m_{L^{\prime}} m_{L}}+W_{L^{\prime} m_{L^{\prime}} ; L m_{L}}\right),
$$

using $\rho_{a}=1$ for distinguishable pions. In the case of $P$-wave scattering of pions, we assume $\delta_{L}=0$ for all $L$ except $L=1$. Hence, the matrix elements of $S-1$ are all zero, except for diagonal entries with $L=1$. This means the matrix $F(S-1)$ has non-zero entries only for columns with $L=1$, so we only need to consider the $3 \times 3$ block involving $L=1$. In all cases, we can reduce the $3 \times 3$ matrix to diagonal form and obtain expressions for $\cot \delta_{1}$ for various $\boldsymbol{d}$ and irreps $\Lambda$, which are summarized in Table 1, defining

$$
w_{l m}=\frac{\mathscr{Z}_{l m}\left(\boldsymbol{s}, \gamma, u^{2}\right)}{\gamma \pi^{3 / 2} u^{l+1}} .
$$




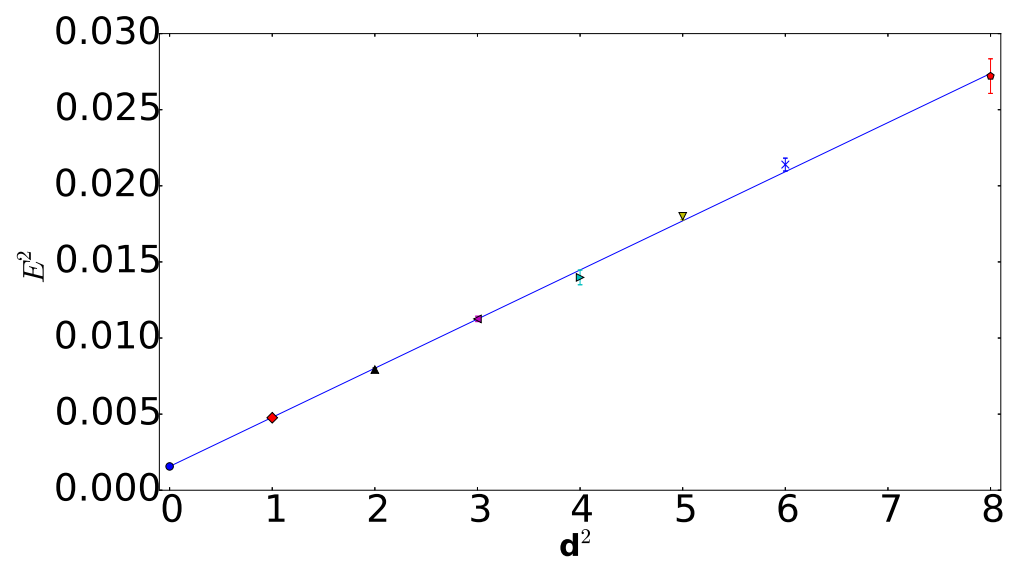

Figure 2: Single pion energies squared $a_{t}^{2} E^{2}$ against $\boldsymbol{d}^{2}$ for our $32^{2} \times 256$ lattice. Fitting to a straight line yields the slope which equals $(\pi /(16 \xi))^{2}$, where $\xi=a_{s} / a_{t}$.

\section{Finite-volume $\pi \pi I=1$ energies}

At rest, the $\rho$ meson appears in the $T_{1 u}^{+}$channel, but for nonzero total momenta, we use results in Ref. [7] to determine which little groups contain the $\rho$. We find that the $\rho$ will appear in the irreps $A_{1}^{+}$and $E^{+}$of $C_{4 v}$ for on-axis total momenta, in the $A_{1}^{+}, B_{1}^{+}$and $B_{2}^{+}$irreps of $C_{2 v}$ for planardiagonal momenta, and $A_{1}^{+}$and $E^{+}$irreps of $C_{3 v}$ for cubic-diagonal momenta. The spectrum of energies from each of these channels can be used to compute the $I=1 \pi \pi P$-wave scattering phase shift, and hence, determine the mass and width of the $\rho$ resonance.

In determining the $\pi \pi$ scattering phase shifts, only energy levels below the inelastic thresholds can be used. In each of the above channels, we include enough two-pion operators of different individual momenta to get a good signal for all states below such thresholds. Our operator construction is described in detail in Ref. [7], and our operator selection procedure and correlator matrix analysis is presented in Ref. [8]. Fig. 1 shows the energies obtained for the interacting $\pi \pi$ levels, compared to the energies of allowed $\pi \pi$ states in the absence of meson-meson interactions. These results are obtained using a $32^{3} \times 256$ anisotropic lattice with quark masses tuned to yield a pion mass around $240 \mathrm{MeV}$. All needed Wick contractions were efficiently evaluated using the stochastic LapH method[6].

\section{1 $P$-wave scattering phase shifts}

To compute the scattering phase shifts using the energies for nonzero total momenta, transformation to the center-of-mass frame is required. Since we are using an anisotropic lattice, energies are measured in terms of the temporal spacing $a_{t}$, while the momenta are given in terms of the larger spatial spacing $a_{s}$. This means changing frames requires a precise knowledge of the renormalized anisotropy $\xi=a_{s} / a_{t}$.

We determine the anisotropy using the dispersion relation of the pion. The energy $E$ of a free 


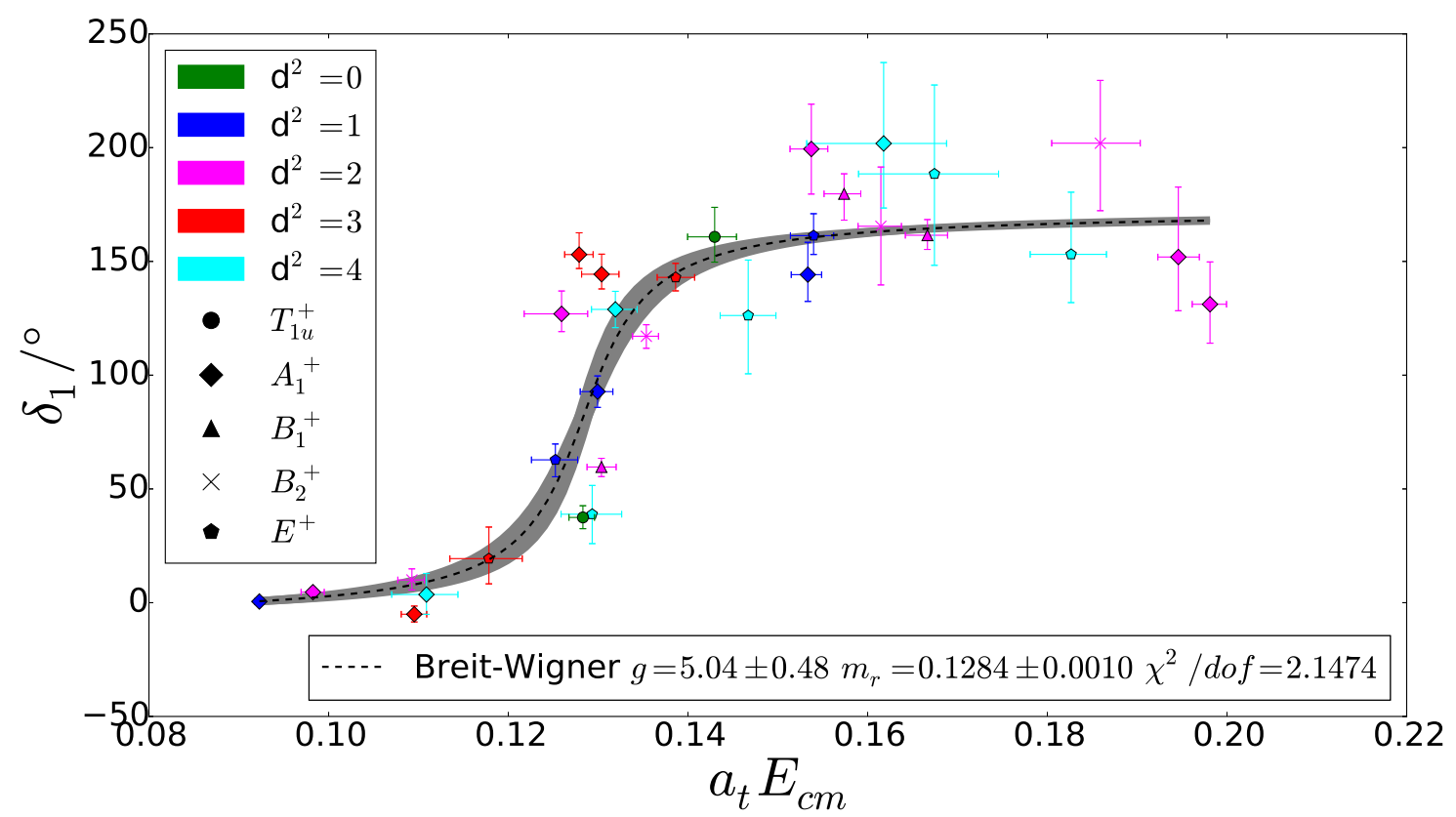

Figure 3: $P$-wave phase shift $\delta_{1}$ again center-of-mass energy $a_{t} E_{\mathrm{cm}}$. Symbol color indicates the $\boldsymbol{d}^{2}$ of the level used, while the symbol shape indicates the irrep. Points which overlap $0^{\circ}$ or $180^{\circ}$ within error are shifted by \pm 180 to ensure continuity of the phase shift. The dashed line indicates the best fit to a Breit-Wigner with the gray band indicating the bootstrap errors in the fit function. These results are still preliminary.

particle of mass $m$ and momentum $\boldsymbol{P}=(2 \pi / L) \boldsymbol{d}$ are related by

$$
\left(a_{t} E\right)^{2}=\left(a_{t} m\right)^{2}+\frac{1}{\xi^{2}}\left(\frac{2 \pi a_{s}}{L}\right)^{2} \boldsymbol{d}^{2}
$$

By evaluating the energies of a particle with different momenta, $\xi$ can be determined. The energies for a single pion for various momenta are shown in Fig. 2. The parameter $\xi$ was fit using a standard least squares fit for each bootstrap resampling.

The energies shown in Fig. 1, as well as the lowest three energies for zero momentum in the $T_{1 u}^{+}$channel obtained in Ref. [8], were used to compute the $\delta_{1}$ phase shift using the expressions given in Table 1. Calculating the phase shift requires not only the energy $E$ of a particular state, but also the mass of the pion $m_{\pi}$ at rest and the renormalized anisotropy $\xi$ to determine $E_{\mathrm{cm}}$, and hence, $\boldsymbol{q}_{\mathrm{cm}}$ and $u$. The formulas in Table 1 yield $\cot \delta_{1}$, which means that care with respect to quadrant must be taken when determining $\delta_{1}$ for measurements on different bootstraps.

Our preliminary results for the $I=1 \pi \pi P$-wave scattering phase shift are shown in Fig. 3 against the center-of-mass energy $a_{t} E_{\mathrm{cm}}$. The mass $m_{r}$ and width $\Gamma$ of the $\rho$ resonance is obtained by fitting the phase shift to a Breit-Wigner form:

$$
\tan \left(\delta_{1}\right)=\frac{\Gamma / 2}{m_{r}-E}+A,
$$


where $A$ parametrizes a slowly-varying background. The width $\Gamma$ is sensitive to the allowed phase space for its decay products, which depends on the pion mass. Since our pion mass is $240 \mathrm{MeV}$, we cannot expect our width determination to agree with experiment. However, the effects of phase space can be reduced by writing the width in terms of a $\rho \pi \pi$ coupling $g$ :

$$
\Gamma=\frac{g^{2}}{48 \pi m_{r}^{2}}\left(m_{r}^{2}-4 m_{\pi}^{2}\right)^{3 / 2} .
$$

The coupling $g$ is expected to be fairly insensitive to the quark mass. Our best-fit values for $m_{r}$ and $\Gamma$, with errors determined by bootstrap resampling, are

$$
a_{t} m_{r}=0.1284 \pm 0.0010 \quad \text { and } \quad g=5.04 \pm 0.48
$$

The location of the resonance is consistent with the value obtained from the spectrum of states in a finite box in the $T_{1 u}^{+}$channel presented in Ref. [8], which yielded a mass $0.1284 \pm 0.0014$. The value of $g$ is slightly low but consistent with its experimental value near 6. Keep in mind that these results are not yet finalized.

\section{Conclusion}

Our progress in calculating the $I=1 \pi \pi P$-wave scattering phase shifts on a large $32^{3} \times 256$ lattice for a light pion mass near $240 \mathrm{MeV}$ was described in this talk. The stochastic LapH method was used to evaluate all needed Wick contractions.

This work was supported by the U.S. National Science Foundation under awards PHY-1306805 and PHY-1318220, and through the NSF TeraGrid/XSEDE resources provided by TACC and NICS under grant number TG-MCA07S017. B. H. is supported by Science Foundation Ireland under Grant No. 11/RFP/PHY3218.

\section{References}

[1] A. Reifman, B.S. DeWitt, R. Newton, Phys. Rev. 101, 877 (1956).

[2] B.S. DeWitt, Phys. Rev. 103, 1565 (1956).

[3] M. Lüscher, Nucl. Phys. B 354, 531 (1991).

[4] K. Rummukainen, S. Gottlieb, Nucl. Phys. B 450, 397 (1995).

[5] C.H. Kim, C.T. Sachrajda, S. Sharpe, Nucl. Phys. B 727, 218 (2005).

[6] C. Morningstar, J. Bulava, J. Foley, K.J. Juge, D. Lenkner, M. Peardon, C.H. Wong, Phys. Rev. D 83, 114505 (2011).

[7] C. Morningstar, J. Bulava, B. Fahy, J. Foley, Y.C. Jhang, K.J. Juge, D. Lenkner, C.H. Wong, Phys. Rev. D 88, 014511 (2013).

[8] C. Morningstar, B. Fahy, Y.C. Jhang, K.J. Juge, D. Lenkner, C.H. Wong, PoS(LATTICE 2014):101 (2014), proceedings of the 32nd International Symposium on Lattice Field Theory (Lattice 2014), 23-28 June 2014, Columbia University, New York, NY, USA. 